\title{
FRIENDS OF THE UNIVERSITY OF IOWA LIBRARIES MEMBERS DURING 1976
}

Patrons

James Montgomery Beck

Mr. and Mrs. Dale M. Bentz, Iowa City, Iowa

Mr. Maury A. Bromsen, Boston, Massachusetts

Martin H. Burge

Mr. and Mrs. Homer L. Calkin, Arlington, Virginia

Mrs. Corinne W. Carter, Keokuk, Iowa

Lawrence O. Cheever

Mrs. Leslie Douglas, Washington, D.C.

Mr. and Mrs. Leslie W. Dunlap, Iowa City, Iowa

Mr. Charles L. Eble, Iowa City, Iowa

Mr. and Mrs. Owen Elliott,

Cedar Rapids, Iowa

Dr. Craig D. Ellyson, Waterloo, Iowa

Mrs. Laurence R. Fairall, Des Moines, Iowa

Mrs. Lewis C. Frank, Jr., New York, New York

Mr. Edwin B. Green, Iowa City, Iowa

Mr. and Mrs. Frank S. Hanlin, Iowa City, Iowa

Mr, and Mrs. William Randolph Hearst, Jr., New York, New York

Hertzberg-New Method, Inc., Jacksonville, Illinois

Institute of Hydraulic Research, Iowa City, Iowa

Mr. Karl Kiedaisch, Jr., Forest Hills, New York
Harvey C. Knowles, Sr.

Dr. R. N. Larimer, Sioux City, Iowa

Bernice Elizabeth Leary

Thomas O. Mabbott

Mrs. Thomas O. Mabbott, New York, New York

Dr. John Martin, Clarinda, Iowa

Miss Hazel Meek, Washington, Iowa

Mrs. Louise R. Noun, Des Moines, Iowa

Mr. Wayne Rawley III, Iowa City, Iowa

Mrs. Gladys W. Richards, Templeton, Massachusetts

Mr. Paul C. Richards, Templeton, Massachusetts

Mrs. Leo W. Schwarz, New York, New York

Irene L. Steidl

Harold Lincoln Thompson

Sara Conn Thompson

The University of Iowa Foundation, Iowa City, Iowa

Mr. Clarence M. Updegraff, San Francisco, California

Mr. Jacob Van Ek, Boulder, Colorado

Mr. and Mrs. Gustave von Groschwitz, New Haven, Connecticut

Mrs. Henry A. Wallace, South Salem, New York

Mr. Henry B. Wallace, West Des Moines, Iowa

Mr. Robert B. Wallace, Washington, D.C.

Dr. Janet B. Wilcox, Iowa City, Iowa

Mr. M. Dean Zenor, Iowa City, Iowa

\section{Sustaining}

Mrs. Julia Bartling, Iowa City, Iowa

Miss May Brodbeck, Iowa City, Iowa

Mr. Robert G. Davis, Kent, Connecticut
Mrs. Barbara Greenleaf, Iowa City, Iowa

Mr. and Mrs. William T. Hageboeck, Iowa City, Iowa 
Mr. Herbert J. Hodges, Davenport, Iowa

Mrs. Frances H. Ingham, Des

Moines, Iowa

Dr. and Mrs. Whealen M. Koontz, Cedar Rapids, Iowa

Mr. Marion R. McCaulley, Austin, Texas

Mr. Kenneth P. MacDonald, Des Moines, Iowa
Dr. Alfred S. Martin, Philadelphia, Pennsylvania

Miss Susan K. Massick, Davenport, Iowa

Mr. and Mrs. J. Richard Wilmeth, Iowa City, Iowa

Zeitlin \& Ver Brugge Booksellers, Los Angeles, California

\section{Contributing}

Dr. and Mrs. George F. Andreasen, Iowa City, Iowa

Mr. and Mrs. Matthew J. Avcin, Jr., Coralville, Iowa

Mr. Ray E. Bazire, Sanibel Island, Florida

Dr. and Mrs. William B. Bean, Galveston, Texas

Dr. George N. Bedell, Iowa City, Iowa

Miss Ardes R. Beisler, New York, New York

Mr. and Mrs. Maurice W. Boatman, Sun City, Arizona

Dr. and Mrs. Michael Bonfiglio, Coralville, Iowa

Mr. and Mrs. Willard L. Boyd, Iowa City, Iowa

Miss Katherine A. Brose, Oakland, California

Mr. Donald C. Bryant, Iowa City, Iowa

Ms. Helen S. Clark, Iowa City, Iowa

Mr. Kenneth E. Colton, Kent, Ohio

Dr. and Mrs. Hunter H. Comly, North Liberty, Iowa

Miss Sarah M. Davis, De Kalb, Illinois

Mr. and Mrs. J. Wayne Deegan, Iowa City, Iowa

Mr. and Mrs. Harry B. Dunlap, Iowa City, Iowa

Mrs. Robert E. Evans, Iowa City, Iowa

Cdr. Don Farran USN (Ret), Rowan, Iowa
Mr. Ronald H. Filinger, Denver, Colorado

Mr. and Mrs. Ronald L. Fingerson, Emporia, Kansas

Mr. and Mrs. John C. Gerber, Guilderland, New York

Mr. and Mrs. Arthur R. Giaquinta, Iowa City, Iowa

Mr. and Mrs. Orville F. Grahame, Worcester, Massachusetts

Miss Linda L. Gunther, Phoenix, Arizona

Mr. and Mrs. Don Guthrie, Iowa City, Iowa

Mr. and Mrs. Charles A. Hale, Iowa City, Iowa

Mr. Reeves Hall, Independence, Iowa

Dr. Hilda J. Hannay, Auburn, Alabama

Mr. John M. Henry, Des Moines, Iowa

Mr. and Mrs. Loren Hickerson, Iowa City, Iowa

Miss Clara Hinton, Iowa City, Iowa

Mr. Delmar C. Homan, Lindsborg, Kansas

Miss Suzanne J. Hosford, Omaha, Nebraska

Mr. and Mrs. Frank Jeffrey, Mason City, Iowa

Miss Grace E. Kemp, Fairfield, Iowa

Mr. and Mrs. Richard M. Kolbet, Iowa City, Iowa

Mr. and Mrs. John S. Koza, Iowa City, Iowa 
Mrs. Jean H. Lloyd-Jones, Iowa

City, Iowa

Mr. Richard Lloyd-Jones, Iowa City, Iowa

Mr. and Mrs. Charles L. Luedtka, Streator, Illinois

Dr. Herberta M. Lundegren, State College, Pennsylvania

McBlain Books, Des Moines, Iowa

Mr. Tom J. McHale, Dallas, Texas

Miss Esther M. McNutt, Rock Island, Illinois

Mrs. Edward F. Misak, Sun City, Arizona

Ms. Jan K. Muhlert, Iowa City, Iowa

Mr. Kenneth Nebenzahl, Chicago, Illinois

Otto Harrassowitz, Wiesbaden, Germany

Mr. Francis J. Paluka, Iowa City, Iowa

Mr. David L. Passmore, Lawrence, Kansas

Mr. and Mrs. Stow Persons, Iowa City, Iowa
Mrs. Ruby B. Powles, Marion, Iowa

Miss Josephine A. Rocca, Sheboygan, Wisconsin

Mr. Malcolm Rohrbough, Iowa City, Iowa

Mr. and Mrs. Hunter Rouse, Sun City, Arizona

Mr. Charles H. Rumsey, Custer, Wisconsin

Miss Marilyn M. Satterlee, Urbana, Illinois

Mr. and Mrs. David G. Schaal, Iowa City, Iowa

Mr. and Mrs. John E. Simmons, Iowa City, lowa

Mrs. Charlotte M. Smith, Newton, Iowa

Mr. and Mrs. Carl S. Vestling, Iowa City, Iowa

Miss Grace Wormer, Iowa City, Iowa Mr. and Mrs. Thomas Yoseloff, Colts Neck, New Jersey

Mrs. Ralph W. Zastrow, Charles City, Iowa

\section{Regular}

Mr. and Mrs. David A. Aamodt, Iowa City, Iowa

Mr. Frank T. Allen, Iowa City, Iowa

Mr. and Mrs. Oren Alt, Iowa City, Iowa

American Antiquarian Society, Worcester, Massachusetts

Mr. Clarence A. Andrews, Iowa City, Iowa

Mr. and Mrs. David Archie, Des Moines, Iowa

Arizona State University Library, Tempe, Arizona

Mr. William G. Asp, St. Paul, Minnesota

Auburn University Library, Auburn, Alabama

Miss Vera J. Bacon, Iowa City, Iowa Miss Frances E. Baker, Sun City, Arizona
Mrs. Gladys S. Bartholow, Washington, lowa

Baylor University Library, Waco, Texas

Mr. and Mrs. Samuel L. Becker, Iowa City, Iowa

Mr. James J. Beilman, Coralville, Iowa

Mr. and Mrs. Arthur Benton, Iowa

City, Iowa

Miss Carol Bentz, Rockford, Illinois

Mr. and Mrs. Dale F. Bentz,

Hacienda Heights, California

Mr. and Mrs. Thomas E. Bentz, Princeton, Massachusetts

Miss Gladys S. Benz, Iowa City, Iowa

Miss Rose Mary Berning, Hudson, Wisconsin

Mr. and Mrs. Richard J. Bloesch, Iowa City, Iowa 
Mr. Robert W. Bolson, Decorah, Iowa

Mr. and Mrs. William Boos, Iowa City, Iowa

Mr. O M Brack, Jr., Tempe, Arizona

British Museum, London, England

Mr. Terrence A. Brooks, Coralville, Iowa

Mr. Larry C. Bruse, Iowa City, Iowa

Mr. and Mrs. Robert E. Buckles, Iowa City, Iowa

Buffalo and Erie County Public Library, Buffalo, New York

Mrs. B. R. Burkhart, Iowa City, Iowa

Mr. and Mrs. Thomas B. Burns, Davenport, Iowa

Mrs. Roger Butterfield, Hartwick, New York

Ms. Susan Graeber Cagni, San Diego, California

Mrs. Aelese L. Carlson, Kensington, Maryland

Carnegie-Mellon University Library, Pittsburgh, Pennsylvania

Mr. Thomas L. Carney, Cedar Rapids, Iowa

Cedar Rapids Public Library, Cedar Rapids, Iowa

Mr. and Mrs. David E. Christ, Iowa City, Iowa

Mr. Jim E. Cole, Iowa City, Iowa

Mr. Carroll Coleman, Iowa City, Iowa

Mrs. Maria M. Collura, West

Liberty, Iowa

Council Bluffs Public Library, Council Bluffs, Iowa

Dr. and Mrs. Dario A. Covi, Louisville, Kentucky

Ms. Joyce Crawford, Cedar Rapids, Iowa

Mr. Robert W. Cryder, Iowa City, Iowa

Mr. William H. Cumberland, Storm Lake, Iowa

Mr. David S. Curry, Iowa City, Iowa

Mr. Charles D. Cuttler, Iowa City, Iowa

Mr. Richard L. Dally, Denver, Colorado
Davenport Public Library, Davenport, Iowa

Des Moines Public Library, Des Moines, Iowa

Miss Shirley B. Dick, Keokuk, Iowa

Mr. Jack W. Dickey, Iowa City, Iowa

Mr. Richard Dilley, Springfield, Illinois

Dr. John M. Dorsey, Highland Park, Michigan

Miss Justine E. Dougherty, Des Moines, Iowa

Mr. Lowell R. Duhrsen, Las Cruces, New Mexico

Mr. Rhodes Dunlap, Iowa City, Iowa

East Carolina University Library, Greenville, North Carolina

Mr. J. M. Edelstein, Washington, D.C.

Mrs. Matilda F. Eliason, Escalon, California

Miss Eva H. Erickson, Iowa City, Iowa

Mr. and Mrs. James G. Ervin, Claremont, California

Miss Signi Falk, Cedar Rapids, Iowa

Mr. Thomas Farrell, Iowa City, Iowa

Mrs. Richard Fisher, Keosauqua, Iowa

Mr. James B. Fitzmaurice, Flagstaff, Arizona

Mr. Richard G. Flamer, Omaha, Nebraska

Miss Frances M. Flanagan, St. Joseph, Missouri

Miss Frances J. Flick, Tucson, Arizona

Miss Helen E. Focht, Iowa City, Iowa

Mr. John W. Forys, Jr., Coralville, Iowa

Mrs. Carolyn Foster, Marshall, Texas

Mr. Gene E. Fries, Marion, Iowa

Miss Nina A. Frohwein, Iowa City, Iowa

Mrs. J. Curtis Frymoyer, Wilton, Iowa

Mrs. Anneliese M. Funke, Iowa City, Iowa 
Mr. W. Lee Garner, Albuquerque, New Mexico

Mr. Lawrence E. Gelfand, Iowa

City, Iowa

Mrs. Evelyn Harter Glick, Darien, Connecticut

Miss Anna A. Goldsberry, New Berlin, Illinois

Mr. Arthur Goldsmith, Jr., Clarksville, Tennessee

Mrs. Linda A. Good, Gowrie, Iowa

Mr. Jerome C. Gorby, South

Charleston, West Virginia

Mr. Lawrence R. Gorman, Iowa City, Iowa

Mr. and Mrs. C. Bruce Gould, Hopewell, New Jersey

Mr. William A. Graf, Iowa City, Iowa

Mr. and Mrs. John E. Grant, Iowa City, Iowa

Mr. and Mrs. Richard S. Green, Iowa City, Iowa

Mrs. Evelyn M. Greenwood, Riverside, California

Grinnell College Library, Grinnell, Iowa

Miss Judith K. Groendyke, Iowa City, Iowa

Miss Betsy L. Hadden, Springfield, Ohio

Mr. Oscar Cornell Hajos, Iowa City, Iowa

Anne-Mieke and John Halbrook, Iowa City, Iowa

Mrs. Antonia W. Hamilton, Iowa City, Iowa

Mrs. Carol K. Harmon, Springfield, Illinois

Mr. and Mrs. Peter J. Hartford, Oxford, Iowa

Mr. J. D. Hartzler, Wellman, Iowa

Mrs. E. H. Hatfield, Waterloo, Iowa

Mr. James Hearst, Cedar Falls, Iowa

Mr. James A. Hedges, Hyattsville, Maryland

Mr. and Mrs. Robert M. Hedges, Hagerstown, Maryland

Mr. James B. Hemesath, Milton, Wisconsin
Miss Judith D. Hendershot, Iowa City, Iowa

Mrs. Forrest Hickman, Iowa City, Iowa

Miss Jane L. Hildebrand, Brunswick, Georgia

Mrs. Richard J. Hingtgen, Marion, Iowa

Mr. O. A. Hitchcock, Iowa City, Iowa

Miss Adeline M. Hoffman, Iowa City, Iowa

Mr. Edwin A. Holtum, Iowa City, Iowa

Mr. Henry G. Horwitz, Iowa City, Iowa

Mr. and Mrs. H. Clark Houghton, Iowa City, Iowa

Mr. David D. Hudson, Coralville, Iowa

Mr. and Mrs. Marion L. Huit, Iowa City, Iowa

Ms. Linda A. Hulbert, Iowa City, Iowa

Mr. Michael D. Hurley, Los Angeles, California

Mr. Timothy N. Hyde, Des Moines, Iowa

Indiana State University Library, Terre Haute, Indiana

Iowa Book \& Supply Company, Iowa City, Iowa

Iowa State University Library, Ames, Iowa

Mr. W. R. Irwin, Iowa City, Iowa

Mrs. Louise F. Janes, Iowa City, Iowa

Dr. and Mrs. Lewis E. January, Iowa City, Iowa

Dr. David C. Jenkins, Williamsburg, Virginia

Miss Rebecca L. Johnson, Iowa City, Iowa

Mr. and Mrs. Howard R. Jones, Solon, Iowa

Mrs. Idamae Jones, Iowa City, Iowa

Mr. John C. Kemmerer, New York, New York

Miss Dorothy Kestel, Coralville, Iowa 
Miss Margaret N. Keyes, Iowa City, Iowa

Mr. and Mrs. John W. Kibby, Santa Maria, California

Miss Dorothy M. Kibler, Iowa City, Iowa

Ms. Sandra Kirshenbaum, San Francisco, California

Mr. and Mrs. Lester L. Kluever, Atlantic, Iowa

Dr. Paul A. Knights, Meadville, Pennsylvania

Mrs. James A. Kohler, Iowa City, Iowa

Mrs. Barbara J. Korn, Alexandria, Virginia

Mrs. Maureen L. Lagomarcino, Arlington, Virginia

Lathrop C. Harper, Inc., New York, New York

Mr. James K. Laurie, Stillwater, Minnesota

Dr. Lillian B. Lawler, Iowa City, Iowa

Mrs. Lesley E. Lawrence, Waverly, Tennessee

Mr. Roger R. Leech, Chicago, Illinois

Mr. and Mrs. Desmond Leigh-Hunt, Santa Barbara, California

Mrs. Esther L. Liffring, Iowa City, Iowa

Wahlert Memorial Library, Dubuque, Iowa

Mrs. Tanja Lorkovic, Iowa City, Iowa

Louisiana State University Library, Baton Rouge, Louisiana

Mr. and Mrs. Robert A. McCown, Iowa City, Iowa

Mr. Daniel T. McDonald, Iowa City, Iowa

Mrs. James McDonald, Cherokee, Iowa

Ms. Mary R. McInroy, Iowa City, Iowa

Mr. Michael Gene Maddigan, Cedar Rapids, Iowa

Miss Gladys E. Manning, Iowa City, Iowa
Miss Lucia A. Marino, Iowa City, Iowa

Mr. and Mrs. William E. Marks, Iowa City, Iowa

Mr. J. David Martin, Iowa City, Iowa

Mr. David E. Miller, Kittanning, Pennsylvania

Mr. Dwight M. Miller, West Branch, Iowa

Minnesota Historical Society, St. Paul, Minnesota

Mrs. Pearl J. Minor, Iowa City, Iowa

Mr. Arthur H. Minters, New York, New York

Mrs. Pearl M. Mirich, Cedar Rapids, Iowa

Mr. and Mrs. Albert R. Mitchell, Canyon, Texas

Mr. and Mrs. Leslie G. Moeller, Iowa City, Iowa

Mr. William M. Moldoff, Nassau, New York

Mrs. Doris E. Moldoff, Nassau, New York

Mr. and Mrs. Russell W. Monson, Iowa City, Iowa

Mr. and Mrs. Ray B. Mossman, Iowa City, Iowa

Mr. and Mrs. Robert W. Munn, Iowa City, Iowa

Muscatine Public Library, Muscatine, Iowa

Miss Virginia A. Myers, Solon, Iowa

Mrs. Ellis H. Newsome, Iowa City, Iowa

Niedersächsische Staats-u. Universitäts-Bibliothek, Göttingen, Germany

Miss Mary E. Noble, Iowa City, Iowa

Mr. Gerald L. Nordquist, Iowa City, Iowa

Dr. Nina J. Noring, Washington, D.C.

North Texas State University

Library, Denton, Texas

Northern Illinois University Library, De Kalb, Illinois

Northwestern University Library, Evanston, Illinois 
Mr. Ferner Nuhn, Claremont, California

Mr. and Mrs. William G. Nusser, Iowa City, Iowa

Mr. Kenneth J. Oberembt, Syracuse, New York

Miss Velva J. Osborn, Iowa City, Iowa

Miss M. Jean Paige, Rockledge, Florida

Pauline Millen, Books, Des Moines, Iowa

Mr. Peter L. Petersen, Canyon, Texas

Mr. Gary M. Peterson, Washington, D.C.

Mrs. Trudy H. Peterson, Washington, D.C.

Mr. Arthur J. Pflughaupt, Iowa City, Iowa

Mr. and Mrs. J. R. Porter, Iowa City, Iowa

Mr. and Mrs. Fred M. Pownall, Iowa City, Iowa

Princeton University Libraries, Princeton, New Jersey

Mr. Keith A. Rageth, Iowa City, Iowa

Mr. Marion E. Rains, Oskaloosa, Iowa

Miss Etta H. Rasmussen, Iowa City, Iowa

Mr. and Mrs. Ronald A. Rayman, Macomb, Illinois

Kenneth W. Rendell, Inc., Newton, Massachusetts

Mr. Earl M. Rogers, Iowa City, Iowa

Mr. Gordon S. Rowley, Kingston, Illinois

Mr. Leland L. Sage, Cedar Falls, Iowa

Mr. and Mrs. Norman Sage, Solon, Iowa

Mr. Harold E. Sargent, Iowa City, Iowa

Mr. R. Keith Sawvel, Montrose, Colorado

Mr. William C. Sayre, Iowa City, Iowa

Dr. Gordon W. Searle, Iowa City, Iowa
Mr. Paul V. Shearer, Washington, Iowa

Mrs. Fred A. Shelton, Laramie, Wyoming

Mr. Harlan L. Sifford, Iowa City, Iowa

Mr. and Mrs. Robert I. Sinn, San Antonio, Texas

Mr. and Mrs. James M. Sjulin, Portland, Oregon

Southern Illinois University Libraries, Carbondale, Illinois

Stanford University Libraries, Stanford, California

State Historical Society of Iowa, Iowa City, Iowa

State Library Commission of Iowa, Des Moines, Iowa

Mr. Geoffrey Steele, Lumberville, Pennsylvania

Ada M. Stoflet

Mr. William A. Stoppel, Des Moines, Iowa

Miss Doris J. Stuck, Iowa City, Iowa

Mr. Curtis W. Stucki, Seattle, Washington

Mr. and Mrs. Dewey B. Stuit, Iowa City, Iowa

Miss Sue A. Summerfield, Minneapolis, Minnesota

Mr. Richard V. Teschner, Iowa City, Iowa

Mr. Thomas H. Thompson, New York, New York

Dr. and Mrs. Franklin H. Top, Iowa City, Iowa

University of Arizona Library, Tucson, Arizona

University of California Library, Berkeley, California

University of California Library, San Diego, La Jolla, California

University of California Library, Santa Barbara, California

University of Chicago Library, Chicago, Illinois

University of Illinois Library, Urbana, Illinois

University of Maryland Library, College Park, Maryland 
University of Michigan Library, Ann Arbor, Michigan

University of Missouri Library, Columbia, Missouri

University of Northern Iowa Library, Cedar Falls, Iowa

University of Pittsburgh, Graduate School of Library and Information Sciences, Pittsburgh, Pennsylvania

University of South Carolina Library, Columbia, South Carolina

University of Toronto Library, Toronto, Ontario, Canada

University of Victoria Library, Victoria, British Columbia, Canada

University of Washington Libraries, Seattle, Washington

University of Wisconsin-LaCrosse Library, LaCrosse, Wisconsin

University of Wisconsin-Milwaukee Library, Milwaukee, Wisconsin

University of Wyoming Library, Laramie, Wyoming

Mrs. George O. Van Allen, Mount Pleasant, Iowa

Mr. Vernon Van Dyke, Iowa City, Iowa

Mr. Edward L. Vaupel, St. Louis, Missouri

Mr. Allan D. Vestal, Iowa City, Iowa

Miss Carol Sue Vogt, Iowa City, Iowa

Mrs. John R. Wachel, Iowa City, Iowa

Mr. Norman J. Waffle, Marion, Iowa
Dr. Margaret A. Waggoner, Chambersburg, Pennsylvania

Mr. James W. Wallace, Des Moines, Iowa

Mr. John J. Walsdorf, Portland, Oregon

Washington State University Library, Pullman, Washington

Washington University Libraries, St. Louis, Missouri

Mr. Stanley Wawzonek, Iowa City, Iowa

Mr. Otha D. Wearin, Hastings, Iowa

Mrs. Eunice Weech, Iowa City, Iowa

Dr. William J. Weiersheuser, Peoria, Illinois

Mr. Theodore N. Weissbuch, Claremont, California

Mr. Dennis C. Wendell, Ames, Iowa

Miss Dorothea Western, Sun City, Arizona

Mr. and Mrs. Frederick Wezeman, Iowa City, Iowa

Miss F. Eugenia Whitehead, Iowa City, Iowa

Mrs. Charles A. Wilhite, Iowa City, Iowa

Mr. Melvin Wolfson, Chappaqua, New York

Mr. Emerson G. Wulling, La Crosse, Wisconsin

Mr. David Zaehringer, San Diego, California

Miss Helen E. Zeller, Iowa City, Iowa 